\title{
The error analysis of the measurement of permittivity of crude oil with the use of interdigital capacitor sensor
}

\author{
Sylwia Przywska* and Melania Suweni Muntini ${ }^{\dagger}$ \\ Department of Physics, Faculty of Mathematics and Natural Science, \\ Institut Teknologi Sepuluh Nopember, Surabaya 61111
}

\begin{abstract}
Abstract-This paper presents the error analysis of the measurement of permittivity of crude oil with the use of a sensor designed and fabricated in our laboratory. The sensor is a interdigital capacitor sensor fabricated with the use of Printed Circuit Board (PCB) technology. This paper also presents the methodology of making the measurement of permittivity of crude oil sample. The discussion of the data obtained during the experiment is presented as well.
\end{abstract}

KEYWORDS: crude oil, interdigital capacitor sensor, error analysis, permittivity http://dx.doi.org/10.12962/

\section{INTRODUCTION}

Crude oil is a naturally occurring petroleum in its unprocessed form. The formation of emulsions during the extraction of crude oil is a serious problem and it is crucial to separate crude oil from the water before further processing. Since the research team from Malaysia [1] proved that microwave heating can significantly enhance the demulsification rate of crude oil the members of Crude Oil Research Team at Technological Institute Sepuluh Nopember Surabaya have been working on heating crude oil by microwave energy. The results of their work were presented in a publication [2].

\section{RESEARCH METHODOLOGY}

The interdigital capacitor sensor (IDC) presented in this publication allows to establish the purity of crude oil by determining its permittivity. The sensor is designed on a printed circuit board (PCB) and it measures the capacity of fluid being under the investigation.

As shown on Figure 1(a) the sensor consists of two electrodes in the xy plane, each electrode consists of $\mathrm{N}$ fingers. In order to obtain relative permittivity of the fluid being under the investigation different potentials $\mathrm{V}_{1}$ and $\mathrm{V}_{2}$ are applied to interdigitated electrodes of the sensor. The electric field lines generated by the sensor penetrate into the material under test and changes the impedance of the sensor. The sensor behaves as a capacitor in which the capacitive reactance becomes a function of system properties.

The relative permittivity $\epsilon_{k}$ of a liquid being under the test

\footnotetext{
*E-MAIL: syl.przywoska@gmail.com

${ }^{\dagger}$ E-MAIL: melania@physics.its.ac.id
}

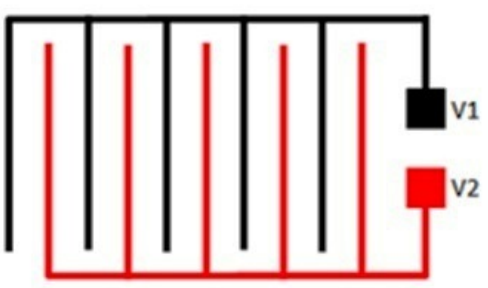

(a) Design of our sensor.

opposite electrode

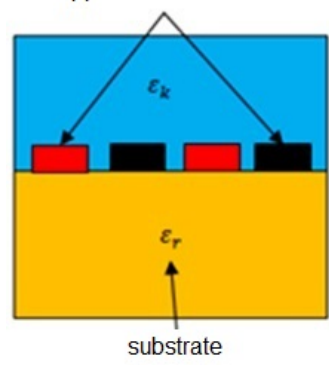

(b) Cross section of IDC

FIG. 1: Design of sensor.

is computed using the following formula [3]:

$$
\epsilon_{k}=\frac{\frac{C_{u c}}{\epsilon_{\circ}}-\epsilon_{r} \frac{k \sqrt{1-\left(\frac{a}{b}\right)^{2}}}{k\left(\frac{a}{b}\right)}}{\frac{k \sqrt{1-\left(\frac{a}{b}\right)^{2}}}{k\left(\frac{a}{b}\right)}+2 \frac{t}{a}}
$$

where: $\mathrm{C}_{u c}=\frac{C_{T O T A L}}{(N-1) L}$ is the unit cell capacitance per length, $\mathrm{C}_{\text {TOT AL }}$ is the result of a measurement of capacitance, $\mathrm{N}$-number of unit cells the capacitor, $\mathrm{L}=5 \mathrm{~cm}$ - length of the electrode fingers [3], $\epsilon_{\circ}$ - dielectric constant in the free space, $\epsilon_{r}=3.6$ [4] - dielectric constant of the substrate (glass fiber reinforced (fiberglass) epoxy resin), $\mathrm{k}()$ - complete elliptic integral of the first kind computed using [5], $\mathrm{t}=(0.20 \pm 0.05) \mathrm{mm}$ - elecrode's thickness, $\mathrm{a}=(2 \pm 0.5) \mathrm{mm}$ - elecrode's width, $\mathrm{b}$ 


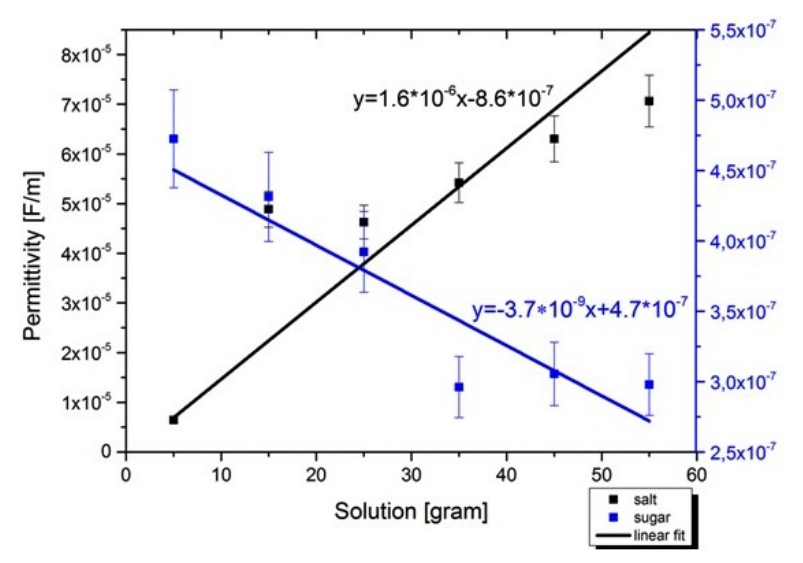

FIG. 2: Obtained changes in relative permittivity for different amount of salt and sugar dissolved in the water.

$=(2.0 \pm 0.2) \mathrm{cm}$ - distance between electrodes.

The permittivity of crude oil is computed by multiplying the relative permittivity $\epsilon_{k}$ by the dielectric constant in the free space $\epsilon_{\circ}$.

The capacitance measurements were performed using the PM 6303 A RCL meter at the frequency of $1 \mathrm{kHz}$. The IDC is directly connected to the instrument via test cable with Kelvin clips. The measurement were performed for one fixed frequency of $1 \mathrm{kHz}$ at room temperature of $25^{\circ}$ and humidity of the air of $84 \%$.

Modus operanti our work is as it follows: (a). The measurement of capacitance for various liquids (like tap water, acetone, salted and sugared water) were made, (b). The capacitances were changed into permittivity values, (c). The calibration of a sensor was made (by comparision of obtained permittivity values with the table ones), (d). The measurement of capacitance for crude oil was made, (e). Analysis and interpretation of obtained data.

\section{RESULT AND ANALYSIS}

The obtained data show the change in permittivity of the fluids according to different amount of salt and sugar added to the water. The trend is raising for the salt (the more salt added to the water the bigger the permittivity is) and decreasing for the sugar (the more sugar added to the water the smaller the permittivity). Both trends are presented on a Figure 2.

The change of the concentration of material (like salt and sugar) in the water causes the change in permittivity of measured materials. The polar nature of the water molecules tends to align with local ion field so it raises the relative permittivity of electrolyte materials when the salt is added, and decreases it when a non-aqueous electrolyte is added like sugar.

The obtained data says that it is possible to distinguish between materials based on the measurement of permittivity with the use of the interdigital capacitor sensor designed and fabricated by us.

After the calibration procedure the measurements of permittivity of crude oil was made. The result is as it follows: 20 $\times 10^{-12} \mathrm{~F} / \mathrm{m}$ with the total relative error of 8 percent. The table values of permittivity of crude oils are between $19.7 \times$ $10^{-12} \mathrm{~F} / \mathrm{m}$ and $21,5 \times 10^{-12} \mathrm{~F} / \mathrm{m}$ for the measurement performed at $1 \mathrm{kHz}$ frequency [?] (depends on the sample). The permittivity obtained in this experiment fits in this brackets.

The total error of the relative permittivity is computed using the finite difference method:

$$
\Delta \epsilon_{k}=\sqrt{\left(\frac{\partial \epsilon_{k}\left(C_{u C}, t, a, b\right)}{\partial C_{u C}} \Delta C_{u C}\right)^{2}+\left(\frac{\partial \epsilon_{k}\left(C_{u C}, t, a, b\right)}{t} \Delta t\right)^{2}+\left(\frac{\partial \epsilon_{k}\left(C_{u C}, t, a, b\right)}{a} \Delta a\right)^{2}}
$$

where: $\Delta C_{u C}$ - is the total error of the unit cell capacitance per length obtained from the formula:

$$
\begin{aligned}
\Delta C_{u C} & =\sqrt{\left(\frac{\partial C_{u C}\left(C_{T O T A L}, L\right)}{\partial C_{T O T A L}} \Delta C_{T O T A L}\right)^{2}+\left(\frac{\partial C_{u C}\left(C_{T O T A L}, L\right)}{\partial C_{L}} \Delta L\right)^{2}} \\
& =\sqrt{\left(\frac{\Delta C_{T O T A L}}{4 L}\right)^{2}+\left(-\frac{C_{T O T A L}}{4 L^{2}}\right)^{2}}
\end{aligned}
$$

where $\triangle C_{T O T A L}$ is a place of least significant number from the capacitance measurement on a RCL meter (the resolution of RCL meter), $\Delta \mathrm{t}$ is the error of a thickness of a electrode $\Delta \mathrm{t}= \pm 0.05 \mathrm{~mm}, \Delta \mathrm{a}$ is the error of the width of a electrode $\Delta \mathrm{a}= \pm$ $0.5 \mathrm{~mm}, \Delta \mathrm{L}$ is the error of the length of the electrodes fingers $\Delta \mathrm{L}= \pm 0,5 \mathrm{~mm}$.

Which leads us to the formula for the total error of the relative permittivity used in this publication:

$$
\Delta \epsilon_{k}=\sqrt{\left(\frac{\frac{1}{\epsilon_{\circ}}}{\frac{k \sqrt{1-\left(\frac{a}{b}\right)^{2}}}{k\left(\frac{a}{b}\right)}+2 \frac{t}{a}} \Delta C_{u C}\right)^{2}+\left(-\frac{2}{a} \frac{\frac{C_{u C}}{\epsilon_{\circ}}-\epsilon_{r} \frac{k \sqrt{1-\left(\frac{a}{b}\right)^{2}}}{k\left(\frac{a}{b}\right)}}{\left(\frac{k \sqrt{1-\left(\frac{a}{b}\right)^{2}}}{k\left(\frac{a}{b}\right)}+2 \frac{t}{a}\right)^{2}} \Delta t\right)^{2}+\left(\frac{2 t}{a^{2}} \frac{\frac{C_{u C}}{\epsilon_{\circ}}-\epsilon_{r} \frac{k \sqrt{1-\left(\frac{a}{b}\right)^{2}}}{k\left(\frac{a}{b}\right)}}{\left(\frac{k \sqrt{1-\left(\frac{a}{b}\right)^{2}}}{k\left(\frac{a}{b}\right)}+2 \frac{t}{a}\right)^{2}} \Delta a\right)^{2}}
$$


In order to obtain the error of permittivity we need to multiply the obtained error of relative permittivity (Eq. 4) by the value of vacuum permittivity $\epsilon_{\circ}$, according to the following equation:

$$
\epsilon=\epsilon_{k} \epsilon_{\circ}
$$

and thus the unit of a permittivity $\epsilon$ is equal to the unit of vacuum permittivity, which means $\frac{F}{m}$.

\section{CONCLUSION}

Summaizing, the error of the permittivity parameter depends on:
1. Accuracy of the measurement of thickness of a electrode $t$, the width of a electrode a, length of the electrodes fingers $\mathrm{L}$.

2. The resolution of RCL meter during the measurement of the capacitance $\mathrm{C}_{\text {TOT AL }}$.

It is also worth to mention that since the sensor is capable of differentiate of various samples of crude oil and its cost of production is relatively low it could be put into a good use in the research of heating crude oil by microwave energy.
[1] H.A. Abdulbari, N.H. Abdurahman, Y. M. Rosli, Wafaa K. Mahmood and H. N.Azhari. Demulsification of petroleum emulsions using microwave separation method

[2] M.S. Mutini, Y.H. Pramono, and Yustiana, Modelling of well drilling heating on crude oil using microwave, AIP Conference Proceedings 1719, 030011, 2016.

[3] A.S. Abu-Abed, and R.G. Lindquist, Progress In Electromagne- tics Research B, 7, 75-87 (2008). doi: 10.2528/PIERB08022901

[4] http://www.kabusa.com/Dilectric-Constants.pdf; http://keisan.casio.com/exec/system/1180573451

[5] Trond Friis, et al., Journal of Dispersion Science and Technology, 19(1), 93-126, January (1998). doi: 10.1080/ 01932699808913163 